\title{
MORITA DUALITY FOR ENDOMORPHISM RINGS
}

\author{
ROBERT W. MILLER AND DARRELL R. TURNIDGE
}

\begin{abstract}
A ring $R$ is said to have a left Morita duality with a ring $S$ if there is an additive contravariant equivalence between two categories of left $R$-modules and right $S$-modules which include all finitely generated modules in ${ }_{R} \mathfrak{M}$ and $\mathfrak{M}_{S}$ respectively and which are both closed under submodules and homomorphic images. We show that for such a ring $R$ the endomorphism ring of every finitely generated projective left $R$-module ${ }_{R} P$ has a left Morita duality with the endomorphism ring of a suitably chosen cofinitely generated injective left $R$-module ${ }_{R} Q$. Specialized to injective cogenerator rings and quasi-Frobenius rings our results yield results of R. L. Wagoner and Rosenberg and Zelinsky giving conditions when the endomorphism ring of a finitely generated projective left module over an injective cogenerator (quasi-Frobenius) ring is an injective cogenerator (quasi-Frobenius) ring.
\end{abstract}

1. Introduction. A ring $R$ is said to have a left Morita duality with a ring $S$ if there is an additive contravariant equivalence between two categories of left $R$-modules and right $S$-modules which include all finitely generated modules in ${ }_{R} \mathfrak{M}$ and $\mathfrak{M}_{S}$ respectively and which are both closed under submodules and homomorphic images. Such a Morita duality exists for $R$ if and only if there exists an injective cogenerator ${ }_{R} U$ for ${ }_{R} \mathfrak{M}$ such that $U_{S}$ is an injective cogenerator for $\mathfrak{M}_{S}$ where $S=$ End $\left({ }_{R} U\right)$ and such that $R \cong$ End $\left(U_{S}\right)$ via the natural mapping ([1], [3], [5], and [6]).

Throughout the following we assume $R$ has such a Morita duality with $S$ via a bimodule ${ }_{R} U_{S}$. Thus by [6] both $R$ and $S$ are semiperfect rings. For $M \in{ }_{R} \mathfrak{M} \quad\left(N \in \mathfrak{M}_{S}\right)$, let $M_{S}^{*}=\operatorname{Hom}_{R}(M, U) \quad\left({ }_{R} N^{*}=\right.$ $\operatorname{Hom}_{S}(N, U)$ ). Then ( $)^{*}$ defines additive contravariant functors from ${ }_{R} \mathfrak{M} \rightarrow \mathfrak{M}_{S}$ and $\mathfrak{M}_{S} \rightarrow{ }_{R} \mathfrak{M}$. A left $R$-module $M$ (right $S$-module $N$ ) is called $U$-reflexive if ${ }_{R} M \cong{ }_{R} M^{* *}\left(N_{S} \cong N_{S}^{* *}\right)$ via the natural mappings. The class of $U$-reflexive modules includes all finitely generated modules in ${ }_{R} \mathfrak{M}\left(\mathfrak{M}_{S}\right)$ and is closed under submodules and homomorphic images.

Received by the editors March 19, 1971.

AMS 1970 subject classifications. Primary 16A49; Secondary 16A50, 16A52.

Key words and phrases. Morita duality, endomorphism rings. 
We show that for each finitely generated projective left $R$-module ${ }_{R} P$ the ring $A=$ End $\left({ }_{R} P\right)$ has a left Morita duality with the ring $B=$ End $\left({ }_{R} Q\right)$ for a suitably chosen cofinitely generated injective left $R$ module ${ }_{R} Q$. Specialized to injective cogenerator rings and quasi-Frobenius rings our results yield results of R. L. Wagoner [10] and Rosenberg and Zelinsky [7].

Throughout the following all rings have identity, all modules are unitary and maps are written opposite the scalars.

2. Results. A module can be shown to be finitely generated if and only if every ascending chain of proper submodules has proper union. Dually, a module is said to be cofinitely generated if every descending chain of nonzero submodules has nonzero intersection, or equivalently if it has finitely generated essential socle. (See [9].)

The following lemma lists several properties of the duality functor ( )* which will be required later.

\subsection{Lemma. Let $M$ be a left $R$-module. Then}

(a) ${ }_{R} M$ is simple if and only if $M_{S}^{*}$ is simple.

(b) ${ }_{R} M$ is finitely generated semisimple if and only if $M_{S}^{*}$ is finitely generated semisimple.

(c) ${ }_{R} M$ is finitely generated if and only if $M_{S}^{*}$ is cofinitely generated.

(d) ${ }_{R} M$ is finitely generated projective if and only if $M_{S}^{*}$ is cofinitely generated injective.

(e) If ${ }_{R} M$ is reflexive, then $\left(\operatorname{Soc}\left({ }_{R} M\right)\right)_{S}^{*} \cong M_{S}^{*} / J\left(M_{S}^{*}\right)$.

Proof. (a) and (b) are left to the reader.

(c) For ${ }_{R} M$ reflexive and $N \subseteq M$ the map given by $N \rightarrow(M / N)^{*}$ yields a lattice anti-isomorphism between the lattice of submodules of ${ }_{R} M$ and $M_{S}^{*}$.

(d) The "only if" follows by Baer's criteria for injectivity. The "if" follows from the fact that for ${ }_{R} P$ finitely generated, ${ }_{R} P$ is projective if and only if ${ }_{R} P$ is $R$-projective. (See [2].)

(e) The socle of ${ }_{R} M$ is the largest semisimple submodule of ${ }_{R} M$. Hence since ${ }_{R} M$ is reflective we have $\left(\operatorname{Soc}\left({ }_{R} M\right)\right)_{S}^{*} \cong M_{S}^{*} / J\left(M_{S}^{*}\right)$, the largest semisimple factor module of $M_{S}^{*}$.

Throughout the remainder of this paper we let ${ }_{R} P$ denote a finitely generated projective left $R$-module and $A=$ End $\left({ }_{R} P\right)$. Since $R$ is semiperfect, ${ }_{R} P / J(P)$ is semisimple and contains a copy of each simple image of ${ }_{R} P$. The following notation will be associated with ${ }_{R} P$.

Let $P_{R}^{\prime}=\operatorname{Hom}_{R}(P, R),{ }_{R} Q=E\left({ }_{R} P / J(P)\right)\left({ }_{R} Q\right.$ is cofinitely generated njective), $B=$ End $\left({ }_{R} Q\right)$, and ${ }_{S} Q^{* \prime}=\operatorname{Hom}_{S}\left(Q^{*}, S\right)$. 
2.2. Proposition. Let the notation be as above. Then

(a) $P_{S}^{*} \cong E\left(Q_{S}^{*} / J\left(Q_{S}^{*}\right)\right)$,

(b) for $X \in \in_{R} \mathfrak{M}, \operatorname{Hom}_{R}(P, X)=0$ if and only if $\operatorname{Hom}_{R}(X, Q)=0$,

(c) $A=\operatorname{End}\left({ }_{R} P\right) \cong \operatorname{End}\left(P_{R}^{\prime}\right) \cong$ End $\left(P_{S}^{*}\right)$.

Proof. (a) $\operatorname{Soc}\left(P_{S}^{*}\right) \cong\left({ }_{R} P / J(P)\right)_{S}^{*}=\left(\operatorname{Soc}\left({ }_{R} Q\right)\right)_{S}^{*} \cong Q_{S}^{*} / J\left(Q_{S}^{*}\right)$ where the isomorphisms follow by (e) of Lemma 2.1 .

(b) Let $0 \neq f \in \operatorname{Hom}(P, X)$ and let $M$ be a simple image of $f(P)$ (and hence of $P)$. Then $\operatorname{Hom}(M, Q) \neq 0$ which implies $\operatorname{Hom}(X, Q) \neq 0$ since ${ }_{R} Q$ is injective. Next let $0 \neq f \in \operatorname{Hom}(X, Q)$. Let $M$ be a simple submodule of $f(X)$ (and hence of $Q$ ). Then $\operatorname{Hom}(P, M) \neq 0$ which implies Hom $(P, X) \neq 0$ since $P$ is projective.

(c) End $\left(P_{R}^{\prime}\right) \cong$ End $\left({ }_{R} P\right) \cong$ End $\left(P_{S}^{*}\right)$ where the second isomorphism is induced by ()$^{*}$ since ${ }_{R} P$ is reflexive.

2.3. Lemma. Let the notation be as above. Then ${ }_{A} P^{\prime} \otimes{ }_{R} Q$ is an injective cogenerator for ${ }_{A} \mathfrak{M}$ with $B \cong$ End $\left({ }_{A} P^{\prime} \otimes_{R} Q\right)$.

Proof. See Corollary 2 to Theorem 3.2 of [8].

2.4. THEOREM. Let $R$ have a left Morita duality with $S$ via a bimodule ${ }_{R} U_{S}$. Let ${ }_{R} P$ be finitely generated projective and let ${ }_{R} Q=E(P / J(P))$. Then the ring $A=$ End $\left({ }_{R} P\right)$ has a left Morita duality with the ring $B=$ End $\left({ }_{R} Q\right)$ via the bimodule ${ }_{A} P^{\prime} \otimes{ }_{R} Q_{B}$.

Proof. $Q_{S}^{*}$ is finitely generated projective. $P_{S}^{*} \cong E\left(Q_{S}^{*} / J\left(Q_{S}^{*}\right)\right)$ is cofinitely generated injective by (d) of Lemma 2.1. So as in Lemma 2.3, $P^{*} \otimes{ }_{S} Q_{B}^{* \prime}$ is an injective cogenerator for $\mathfrak{M}_{B}$ with $A \cong$ End $\left(P^{*} \otimes_{S} Q_{B}^{* \prime}\right)$. But $_{A} P^{*} \otimes{ }_{S} Q_{B}^{* \prime} \cong \operatorname{Hom}_{S}\left(Q_{S}^{*}, P_{S}^{*}\right) \cong \operatorname{Hom}_{R}\left({ }_{R} P,{ }_{R} Q\right) \cong{ }_{A} P^{\prime} \otimes{ }_{R} Q_{B}$. The middle isomorphism follows by ()$^{*}$ since everything in sight is reflexive. Thus ${ }_{A} P^{\prime} \otimes{ }_{R} Q_{B}$ yields the required Morita duality.

3. Applications. A ring $R$ is called an injective cogenerator ring if both ${ }_{R} R$ and $R_{R}$ are injective cogenerators, i.e. if ${ }_{R} R_{R}$ yields a Morita duality of $R$ with itself. An injective cogenerator ring which is left (equivalently right) Artinian is called quasi-Frobenius.

Our results show that the endomorphism ring of a finitely generated projective right or left $R$-module has both a left and a right Morita duality if $R$ is an injective cogenerator ring. In general the endomorphism ring of a finitely generated projective right $R$-module over a quasi-Frobenius ring can fail to be quasi-Frobenius [7].

R. L. Wagoner calls a module ${ }_{R} M$ an $R Z$ module if it has the property that every simple homomorphic image of ${ }_{R} M$ is isomorphic to a simple submodule of ${ }_{R} M$. Using the notation of the preceding section one has that for ${ }_{R} P$ a finitely generated projective left $R$-module with $R$ an injective 
cogenerator ring, ${ }_{R} P$ is an $R Z$ module if and only if ${ }_{R} Q$ is similar to ${ }_{R} P$. Two modules are said to be similar if each is isomorphic to a direct summand of a finite direct sum of copies of the other. Specializing the above theorem to this setting we obtain the following results of $\mathbf{R}$. L. Wagoner [10] and Rosenberg and Zelinsky [7].

3.1. Corollary. Let $R$ be an injective cogenerator ring. Let ${ }_{R} P$ be a finitely generated projective left $R Z$ module. Then $A=$ End $\left({ }_{R} P\right)$ is an injective cogenerator ring.

Proof. Let ${ }_{R} P$ be finitely generated projective. Since similar modules have Morita equivalent endomorphism rings [4] and $R$ is semiperfect we may assume ${ }_{R} P$ is a direct sum of nonisomorphic indecomposable projective (and injective) submodules. Via this reduction if ${ }_{R} P$ is an $R Z$ module ${ }_{R} Q \cong E(P / J(P)) \cong{ }_{R} P$. Thus ${ }_{A} P^{\prime} \otimes{ }_{R} P_{A} \cong{ }_{A} A_{A}$ yields a Morita duality for $A$ with itself. Thus $A$ is an injective cogenerator ring.

3.2. CoROllary. Let $R$ be a quasi-Frobenius ring. Let ${ }_{R} P$ be a finitely generated projective left $R Z$ module. Then $A=$ End $\left({ }_{R} P\right)$ is quasi-Frobenius.

Proof. This follows from Corollary 3.1 and the fact that the endomorphism ring of a finitely generated projective left module over an Artinian ring is Artinian.

\section{REFERENCES}

1. G. Azumaya, A duality theory for injective modules, Amer. J. Math. 81 (1959), 249-278. MR 21 \#5662.

2. —, M-projective and M-injective modules, Ring Theory Symposium Notes, University of Kentucky, Lexington, Ky., 1970.

3. K. Morita, Duality for modules and its applications to the theory of rings with minimum condition, Sci. Rep. Tokyo Kyoiku Daigaku Sect. A 6 (1958), 83-142. MR 20 \#3183.

4. B. J. Mueller, Dominant dimension of semi-primary rings, Crelles J. 232 (1968), 173-179.

5. - Linear compactness and Morita duality, J. Algebra 16, (1970), 60-66.

6. B. Osofsky, A generalization of quasi-Frobenius rings, J. Algebra 4 (1966), 373-387. MR 34 \#4305.

7. A. Rosenberg and D. Zelinsky, Annihilators, Portugal. Math. 20 (1961), 53-65. MR 24 \#A1296.

8. F. L. Sandomierski, On QF-3 rings (to appear).

9. P. Vámos, The dual of the notion of "finitely generated," J. London Math. Soc. 43 (1968), 643-646. MR 40 \#1425.

10. R. L. Wagoner, Cogenerator endomorphism rings, Proc. Amer. Math. Soc. 28 (1971), 347-351.

Department of Mathematics, Kent State University, Kent, Ohio 44242 